\title{
Reconfigurable Antenna Arrays with Multiple Requirements: A Versatile 3D Approach
}

\author{
Massimiliano Comisso, Giulia Buttazzoni, and Roberto Vescovo \\ Department of Engineering and Architecture, University of Trieste, Via A. Valerio 10, 34127 Trieste, Italy \\ Correspondence should be addressed to Massimiliano Comisso; mcomisso@units.it
}

Received 8 February 2017; Accepted 9 April 2017; Published 27 April 2017

Academic Editor: Yuan Yao

Copyright (C) 2017 Massimiliano Comisso et al. This is an open access article distributed under the Creative Commons Attribution License, which permits unrestricted use, distribution, and reproduction in any medium, provided the original work is properly cited.

\begin{abstract}
This paper proposes a deterministic method for the 3D synthesis of antenna arrays that jointly accounts for far-field pattern reconfigurability, polarization setting, dynamic range ratio reduction, and near-field control. The conceived algorithm, which generalizes some existing solutions, relies on a weighted cost function, whose iterative minimization is accomplished by properly derived closed-form expressions. This feature, combined with the possibility of selecting the weighting parameters, provides a fast and versatile approach, whose capabilities are numerically checked by considering different synthesis problems and array structures in the presence of mutual coupling.
\end{abstract}

\section{Introduction}

The exploitation of antenna arrays for generating radiation patterns with desired characteristics has represented since decades a classic research field of applied electromagnetism. Accordingly, a huge number of synthesis methods have been developed for enabling an array to produce patterns satisfying constraints on the steering of the main lobe(s), on the sidelobe level (SLL), and on the depth of the possible null regions $[1,2]$. Beside the shape of the pattern, the synthesis process has often to account for further requirements dependent on the specific scenario in which the array has to operate.

The amplitude of the excitations represents one of the quantities that is usually subject to additional constraints. In fact, the control of the dynamic range ratio (DRR), that is, the ratio between the maximum and the minimum excitation amplitude of the array elements, allows the practical realization of noncomplex feeding networks, in which the number of power dividers may be kept low or the design of the feeding striplines may be simplified. To jointly match the pattern and feeding network constraints, many solutions have been proposed, some allowing selecting a specified value for each excitation amplitude [3], others reducing the DRR [4-6], and still others enabling phase-only control $[7,8]$.
Reconfigurability represents a second significant capability that may be requested to antenna system designers. Current communication systems might in fact have to accomplish multitask missions, in which the pattern must be reshaped by keeping the excitation amplitudes of the elements constant and modifying the sole excitation phases. Antenna systems providing this functionality are referred to as reconfigurable arrays and their actual implementation may sometimes have to account for the additional requirement of DRR reduction $[9,10]$.

One of the common aspects of forthcoming communication systems, such as last generation radars, CubeSat spacecrafts, gigabit-WiFi routers, and $5 \mathrm{G}$ devices, is their shift towards higher frequencies, characterized by stronger signal attenuations and more severe propagation conditions. In this context, the benefits achievable by a careful control of the polarization in terms of fading mitigation and mismatch reduction may become determinant for the viability of a given technology. For this reason, array synthesis algorithms specifically accounting for linear [10-12] and circular or elliptical polarization $[13,14]$ have been recently developed. Some of these methods also combine polarization control with DRR reduction and even with reconfigurability [10], thus allowing addressing more general scenarios. 
During the antenna deployment operations, engineers may have to deal with undesired scattering effects due to close conducting obstacles or with nearby interfering equipment. In these cases, a reduction of the electric near-field amplitude in a prescribed region may be imposed by mandatory regulations or realizability constraints. This problem has been analyzed in $[15,16]$, focusing on the minimization of the near-field in a region lying in the proximity of the antenna, and further deepened in $[17,18]$, by adding DRR control.

The above brief overview of the typical constraints and of the presented solutions for antenna array synthesis puts into evidence that the combination of different requirements may give rise to a wide set of possible problems. In such a scenario, the availability of a general approach, able to manage the manifold situations that may appear in antenna array synthesis, may represent a desirable advance.

To address this issue, this paper proposes a fast and versatile algorithm for the $3 \mathrm{D}$ pattern synthesis of antenna arrays of arbitrary geometry that enables jointly considering DRR reduction, far-field reconfigurability, polarization control, and near-field reduction. The developed method generalizes some existing algorithms by defining a weighted cost function, which is iteratively minimized using expressions derived in closed form. The performance of the conceived solution is numerically investigated by considering different synthesis problems and array structures in which the mutual coupling effects are carefully taken into account.

The paper is organized as follows. Section 2 describes the analyzed problem. Section 3 presents the development of the algorithm. Section 4 discusses the numerical results. Section 5 remarks the most relevant conclusions.

Throughout the paper, the following notations are used: $(\cdot)^{*}$ denotes the complex conjugate, $j$ identifies the imaginary unit, $\mathfrak{R}(\cdot)$ denotes the real part, $(\cdot)^{T}$ represents the transpose operator, $\mathbf{0}_{J \times K}$ identifies the null matrix of dimensions $J \times K$, and $\theta \in[0, \pi]$ and $\phi \in[-\pi, \pi[$ denote the zenith and azimuth angles, respectively.

\section{Problem Formulation}

2.1. Array Patterns. With reference to a spherical coordinate system $O(r, \theta, \phi)$ in the $3 \mathrm{D}$ space, consider an antenna array of $N$ elements, where the position of the $n$th element is described by the vector $\mathbf{r}_{n}=r_{n} \sin \theta_{n} \cos \phi_{n} \widehat{\mathbf{x}}+$ $r_{n} \sin \theta_{n} \sin \phi_{n} \widehat{\mathbf{y}}+r_{n} \cos \theta_{n} \widehat{\mathbf{z}}$, in which $\widehat{\mathbf{x}}, \widehat{\mathbf{y}}$, and $\widehat{\mathbf{z}}$ represent the unit vectors of the Cartesian coordinate axes $x, y$, and $z$, respectively. Besides, consider a set $\mathbf{a}=\left\{\mathbf{a}_{l}\right\}_{l=1}^{L}$ of $L$ column vectors. The $l$ th element of $\mathbf{a}, \mathbf{a}_{l}=\left[a_{1 l}, \ldots, a_{N l}\right]^{T}$, represents the complex array excitations that generate the $l$ th electric farfield vector in the direction $\Omega=(\theta, \phi)$ :

$$
\mathbf{F}\left(\mathbf{a}_{l} ; \Omega\right)=F_{\theta}\left(\mathbf{a}_{l} ; \Omega\right) \hat{\boldsymbol{\theta}}+F_{\phi}\left(\mathbf{a}_{l} ; \Omega\right) \hat{\boldsymbol{\phi}},
$$

where $F_{\theta}\left(\mathbf{a}_{l} ; \Omega\right)$ and $F_{\phi}\left(\mathbf{a}_{l} ; \Omega\right)$ are the electric far-field components along the standard unit vectors $\widehat{\boldsymbol{\theta}}$ and $\widehat{\boldsymbol{\phi}}$, respectively. In particular, these components may be expressed as

$$
\begin{aligned}
& F_{\theta}\left(\mathbf{a}_{l} ; \Omega\right)=\sum_{n=1}^{N} a_{n l} \widetilde{\mathscr{F}}_{n}(\Omega) e^{j \kappa \mathbf{r}_{n} \cdot \widehat{\mathbf{r}}(\Omega)}=\sum_{n=1}^{N} a_{n l} \widetilde{f}_{n}(\Omega), \\
& F_{\phi}\left(\mathbf{a}_{l} ; \Omega\right)=\sum_{n=1}^{N} a_{n l} \widehat{\mathscr{F}}_{n}(\Omega) e^{j \kappa \mathbf{r}_{n} \cdot \widehat{\mathbf{r}}(\Omega)}=\sum_{n=1}^{N} a_{n l} \widehat{f}_{n}(\Omega),
\end{aligned}
$$

where $\widetilde{\mathscr{F}}_{n}(\Omega)$ and $\widehat{\mathscr{F}}_{n}(\Omega)$ are the components along $\widehat{\boldsymbol{\theta}}$ and $\widehat{\phi}$, respectively, of the electric far-field pattern of the $n$th array element, $\kappa$ is the wavenumber, $\widehat{\mathbf{r}}(\Omega)=\sin \theta \cos \phi \widehat{\mathbf{x}}+$ $\sin \theta \sin \phi \widehat{\mathbf{y}}+\cos \theta \widehat{\mathbf{z}}$ is the unit vector of the direction of observation, $\widetilde{f}_{n}(\Omega)=\widetilde{\mathscr{F}}_{n}(\Omega) e^{j \kappa \mathbf{r}_{n} \cdot \widehat{\mathbf{r}}(\Omega)}$, and $\widehat{f}_{n}(\Omega)=$ $\widehat{\mathscr{F}}_{n}(\Omega) e^{j \kappa \mathbf{r}_{n} \cdot \widehat{\mathbf{r}}(\Omega)}$ for $n=1, \ldots, N$. In the $3 \mathrm{D}$ space surrounding the antenna, the array excitation vector $\mathbf{a}_{l}$ also generates, at the point $\mathbf{r}=r \sin \theta \cos \phi \widehat{\mathbf{x}}+r \sin \theta \sin \phi \widehat{\mathbf{y}}+r \cos \theta \widehat{\mathbf{z}}$, the $l$ th electric near-field vector:

$$
\mathbf{E}\left(\mathbf{a}_{l} ; \mathbf{r}\right)=\sum_{n=1}^{N} a_{n l} \mathbf{E}_{n}(\mathbf{r}),
$$

where $\mathbf{E}_{n}(\mathbf{r})$ is the electric near-field produced in $\mathbf{r}$ by the excitation vector $\mathbf{e}_{n}=[0, \ldots, 0,1,0, \ldots, 0]^{T}$, having unity in the $n$th position. Observe that the formulation in $(2 \mathrm{a})$, (2b), and (3) includes the mutual coupling between the array elements. In fact, as discussed in $[13,14]$, the single-element patterns $\widetilde{\mathscr{F}}_{n}(\Omega), \widehat{\mathscr{F}}_{n}(\Omega)$, and $\mathbf{E}_{n}(\mathbf{r})$ can be evaluated by a suitable electromagnetic software using $\mathbf{e}_{n}$ as the excitation vector, that is, considering a unity-voltage zero-phase feed for the $n$th element and a zero-voltage feed for the remaining $N-1$ elements. This approach, which exploits the linear relationships in (2a), (2b), and (3) between the array and the element patterns, enables obtaining $\widetilde{\mathscr{F}}_{n}(\Omega), \widehat{\mathscr{F}}_{n}(\Omega)$, and $\mathbf{E}_{n}(\mathbf{r})$ not as isolated element patterns but as equivalent element patterns in the presence of the rest of the array.

2.2. Synthesis Requirements. The here formulated problem addresses a really general scenario, in which the electric farfield and near-field patterns defined in (2a), (2b), and (3) have to satisfy five different synthesis requirements. First, for $l=1, \ldots, L$, the amplitude of $F_{\theta}\left(\mathbf{a}_{l} ; \Omega\right)$ has to approximate a positive function normalized to unity $\mathscr{M}_{l}(\Omega)$, which specifies the desired pattern shape and the corresponding $3 \mathrm{~dB}$ main lobe region $\Theta_{l} \subset \Theta=[0, \pi] \times[-\pi, \pi[$. Second, for $l=$ $1, \ldots, L, \mathbf{F}\left(\mathbf{a}_{l} ; \Omega\right)$ has to be polarized in $\Theta_{l}$ according to a pair of coefficients $(\delta, \xi)$, with $\delta \geq 0$ and $-\pi \leq \xi \leq \pi$, that is, $F_{\theta}\left(\mathbf{a}_{l} ; \Omega\right)=F_{\phi}\left(\mathbf{a}_{l} ; \Omega\right) \delta e^{j \xi}$. Third, the amplitude of $\mathbf{E}\left(\mathbf{a}_{l} ; \mathbf{r}\right)$ has to be reduced in a prescribed region $\mathbf{R}$ located in the nearfield zone. Fourth, the $L$ amplitude patterns $\left\{F_{\theta}\left(\mathbf{a}_{l} ; \Omega\right)\right\}_{l=1}^{L}$ have to be synthesized with the same distribution of the excitation amplitudes, that is, $\left|a_{n 1}\right|=\cdots=\left|a_{n L}\right|=\alpha_{n}$, for $n=1, \ldots, N$, so as to enable pattern reconfigurability by phase-only control. Fifth, the DRR of the excitations, defined as $\operatorname{DRR}(\mathbf{a}) \triangleq \alpha_{\max } / \alpha_{\text {min }}$ with $\alpha_{\max }=\max _{n} \alpha_{n}$ and $\alpha_{\text {min }}=$ $\min _{n} \alpha_{n}$, has to be reduced to simplify the realization of the 
array feeding network. According to these requirements, the addressed problem may be hence mathematically formulated as that of finding a set of excitation vectors $\mathbf{a}$, that is, a vector $\boldsymbol{\alpha}=\left[\alpha_{1}, \ldots, \alpha_{N}\right]$ and an $N \times L$ matrix $\psi=\left[\psi_{n l}\right]$, such that

$$
\begin{gathered}
\left|F_{\theta}\left(\mathbf{a}_{l} ; \Omega\right)\right| \sim \mathscr{M}_{l}(\Omega), \quad \Omega \in \Theta, l=1, \ldots, L, \\
F_{\theta}\left(\mathbf{a}_{l} ; \Omega\right)=F_{\phi}\left(\mathbf{a}_{l} ; \Omega\right) \delta e^{j \xi}, \quad \Omega \in \Theta_{l}, l=1, \ldots, L, \\
\left|\mathbf{E}\left(\mathbf{a}_{l} ; \mathbf{r}\right)\right| \text { is reduced, } \quad \mathbf{r} \in \mathbf{R}, l=1, \ldots, L, \\
\left|a_{n, l}\right|=\alpha_{n}, \quad l=1, \ldots, L, n=1, \ldots, N, \\
\frac{\alpha_{\max }}{\alpha_{\min }} \text { is reduced. }
\end{gathered}
$$

\section{Synthesis Algorithm}

The above formulated problem may be solved by first introducing the following cost function:

$\mathscr{C}(\mathbf{a})$

$$
\begin{aligned}
& =\sum_{l=1}^{L} \int_{\Theta}|| F_{\theta}\left(\mathbf{a}_{l} ; \Omega\right)\left|-\frac{\mathscr{M}_{l}(\Omega)}{w_{l}(\Omega)}\right|^{2} w_{l}(\Omega) \mathrm{d} \Omega \\
& \quad+c_{P} \sum_{l=1}^{L} \int_{\Theta_{l}}\left|F_{\theta}\left(\mathbf{a}_{l} ; \Omega\right)-F_{\phi}\left(\mathbf{a}_{l} ; \Omega\right) \delta e^{j \xi}\right|^{2} v_{l}(\Omega) \mathrm{d} \Omega \\
& \quad+c_{E} \sum_{l=1}^{L} \int_{\mathbf{R}}\left|\mathbf{E}\left(\mathbf{a}_{l} ; \mathbf{r}\right)\right|^{2} u_{l}(\mathbf{r}) \mathrm{d} \mathbf{r} \\
& \quad+c_{D} \sum_{m, n=1}^{N}\left(\alpha_{n}-\alpha_{m}\right)^{2}
\end{aligned}
$$

where $w_{l}(\Omega), v_{l}(\Omega)$, and $u_{l}(\mathbf{r})$ are positive weight functions, $c_{P}, c_{E}$, and $c_{D}$ are nonnegative weights, and $\mathrm{d} \Omega=\mathrm{d} \theta \mathrm{d} \phi$. This formulation generalizes the problems considered in $[9,14,17]$, which become particular cases of (5). More precisely, for $L=$ 1 and $c_{P}=0$, one obtains the scenario addressed in [17], for $c_{P}=c_{E}=0$, and restricting the problem to the sole azimuth domain, one obtains the scenario analyzed in [9], while, for $L=1$ and $c_{E}=0$, one finally obtains the scenario considered in [14]. In the here considered general case, minimizing the first term in (5) allows the amplitude of each pattern $F_{\theta}\left(\mathbf{a}_{l} ; \Omega\right)$ to approximate the corresponding $\mathscr{M}_{l}(\Omega) / w_{l}(\Omega)$ function, where $w_{l}(\Omega)$ may be conveniently selected close to unity in $\Theta_{l}$ and greater than unity in $\Theta-\Theta_{l}$, in order to obtain a good approximation of each desired pattern in the main lobe region while properly reducing the side-lobe level outside it. Minimizing the second term in (5) allows one to impose the desired polarization for each far-field vector by deriving a pattern $F_{\phi}\left(\mathbf{a}_{l} ; \Omega\right)$ approximately scaled by $1 / \delta$ and with a phase shift $-\xi$ with respect to $F_{\theta}\left(\mathbf{a}_{l} ; \Omega\right)$. Minimizing the third term of $\mathscr{C}(\mathbf{a})$ allows the reduction of the amplitude of each electric near-field vector in $\mathbf{R}$, while, finally, minimizing the last term allows one to reduce the DRR of the excitations.

The power synthesis problem in (5) can be reformulated as a field synthesis one by introducing a set of $L$ auxiliary phase functions $\Phi=\left\{\Phi_{l}(\Omega)\right\}_{l=1}^{L}$, so as to replace each term $\mathscr{M}_{l}(\Omega)$ by $\mathscr{M}_{l}(\Omega) e^{j \Phi_{l}(\Omega)}$. Then, the problem of minimizing (5) becomes equivalent to that of finding the sets a and $\Phi$ that minimize the functional:

$$
\begin{aligned}
& \mathscr{I}(\mathbf{a} ; \mathbf{\Phi}) \\
& =\sum_{l=1}^{L} \int_{\Theta}\left|F_{\theta}\left(\mathbf{a}_{l} ; \Omega\right)-\frac{\mathscr{M}_{l}(\Omega) e^{j \Phi_{l}(\Omega)}}{w_{l}(\Omega)}\right|^{2} w_{l}(\Omega) \mathrm{d} \Omega \\
& \quad+c_{P} \sum_{l=1}^{L} \int_{\Theta_{l}}\left|F_{\theta}\left(\mathbf{a}_{l} ; \Omega\right)-F_{\phi}\left(\mathbf{a}_{l} ; \Omega\right) \delta e^{j \xi}\right|^{2} v_{l}(\Omega) \mathrm{d} \Omega \\
& \quad+c_{E} \sum_{l=1}^{L} \int_{\mathbf{R}}\left|\mathbf{E}\left(\mathbf{a}_{l} ; \mathbf{r}\right)\right|^{2} u_{l}(\mathbf{r}) \mathrm{d} \mathbf{r} \\
& \quad+c_{D} \sum_{m, n=1}^{N}\left(\alpha_{n}-\alpha_{m}\right)^{2} .
\end{aligned}
$$

Substituting (2a), (2b), and (3) in (6) and setting $a_{n l}=\alpha_{n} e^{j \psi_{n l}}$ for $n=1, \ldots, N$ and $l=1, \ldots, L$ to impose the requirement concerning phase-only control, after some manipulations one can rewrite $\mathscr{I}(\mathbf{a} ; \Phi)$ as

$$
\begin{aligned}
& \mathscr{I}(\mathbf{a} ; \boldsymbol{\Phi})=\sum_{l=1}^{L}\left[\sum_{m, n=1}^{N} \alpha_{n} \alpha_{m} e^{j\left(\psi_{n l}-\psi_{m l}\right)} A_{l m n}\right. \\
& \left.-2 \sum_{n=1}^{N} \alpha_{n} \mathfrak{R}\left(e^{j \psi_{n l}} g_{n l}^{*}\right)+\Upsilon_{l}^{2}\right]+2 c_{D}\left[N \sum_{n=1}^{N} \alpha_{n}^{2}\right. \\
& \left.-\left(\sum_{n=1}^{N} \alpha_{n}\right)^{2}\right],
\end{aligned}
$$

where

$$
\begin{aligned}
& A_{l m n}=\int_{\Theta} \widetilde{f}_{n}^{*}(\Omega) \widetilde{f}_{m}(\Omega) w_{l}(\Omega) \mathrm{d} \Omega \\
& \quad+c_{P} \int_{\Theta_{l}}\left\{-\delta\left[\widehat{f}_{n}^{*}(\Omega) \tilde{f}_{m}(\Omega) e^{-j \xi}\right.\right. \\
& \left.\quad+\widetilde{f}_{n}^{*}(\Omega) \widehat{f}_{m}(\Omega) e^{j \xi}\right]+\widetilde{f}_{n}^{*}(\Omega) \tilde{f}_{m}(\Omega)+\delta^{2} \widehat{f}_{n}^{*}(\Omega) \\
& \left.\quad \cdot \widehat{f}_{m}(\Omega)\right\} v_{l}(\Omega) \mathrm{d} \Omega+c_{E} \int_{\mathbf{R}} \mathbf{E}_{n}(\mathbf{r}) \cdot \mathbf{E}_{m}^{*}(\mathbf{r}) u_{l}(\mathbf{r}) \mathrm{d} \mathbf{r}, \\
& g_{n l}=\int_{\Theta} \tilde{f}_{n}^{*}(\Omega) \mathscr{M}_{l}(\Omega) e^{j \Phi_{l}(\Omega)} \mathrm{d} \Omega, \\
& \Upsilon_{l}^{2}=\int_{\Theta} \frac{\mathscr{M}_{l}^{2}(\Omega)}{w_{l}(\Omega)} \mathrm{d} \Omega .
\end{aligned}
$$

The functional in (7) can be minimized by adopting an iterative approach in which each iteration is subdivided into two parts. In the first part, given $\Phi, \mathscr{I}(\mathbf{a} ; \Phi)$ is minimized with respect to the excitation amplitudes (common for the $L$ synthesized patterns) and to the excitation phases (different for the $L$ synthesized patterns). The $N$ amplitudes and the 
$L \times N$ phases are evaluated one at a time. To this aim, one may notice that $A_{l m n}=A_{l n m}^{*}$; thus (7) can be elaborated to put into evidence its dependence on the generic amplitude $\alpha_{p}$ and on the row vector of phases $\psi_{p}=\left[\psi_{p 1}, \ldots, \psi_{p L}\right]$ as

$$
\mathscr{I}(\mathbf{a} ; \boldsymbol{\Phi})=\beta_{p} \alpha_{p}^{2}+2 \gamma_{p}\left(\psi_{p}\right) \alpha_{p}+\omega_{p}
$$

where

$$
\begin{aligned}
\beta_{p}= & \sum_{l=1}^{L} A_{l p p}+2 c_{D}(N-1), \\
\gamma_{p}\left(\psi_{p}\right)= & \sum_{l=1}^{L}\left\{\left|T_{p l}\right| \cos \left[\psi_{p l}-\arg \left(T_{p l}\right)\right]\right\} \\
& +2 c_{D} \sum_{\substack{n=1 \\
n \neq p}}^{N} \alpha_{n}, \\
T_{p l}= & \sum_{\substack{n=1 \\
n \neq p}}^{N} \alpha_{n} e^{j \psi_{n l}} A_{l p n}-g_{p l}
\end{aligned}
$$

and $\omega_{p}$ is a term independent of $\alpha_{p}$ and $\psi_{p}$. Now, imposing that the derivative of (9) with respect to $\alpha_{p}$ be zero and that $\gamma_{p}\left(\psi_{p}\right)$ in (10b) be minimum with respect to $\psi_{p l}$, one obtains the amplitude and the phase minimizing $\mathscr{I}(\mathbf{a} ; \boldsymbol{\Phi})$ as

$$
\begin{gathered}
\alpha_{p}=\frac{1}{\beta_{p}}\left(\sum_{l=1}^{L}\left|T_{p l}\right|+2 c_{D} \sum_{\substack{n=1 \\
n \neq p}}^{N} \alpha_{n}\right), \\
\psi_{p l}=\arg \left(T_{p l}\right)+\pi, \quad l=1, \ldots, L
\end{gathered}
$$

for $p=1, \ldots, N$.

In the second part of each iteration, given a, $\mathscr{I}(\mathbf{a} ; \Phi)$ is minimized with respect to $\Phi$. This objective is achieved by substituting (8b) into (7) and putting into evidence its dependence on the generic phase function $\Phi_{l}(\Omega)$, thus obtaining

$$
\begin{aligned}
& \mathscr{I}(\mathbf{a} ; \boldsymbol{\Phi})=\eta_{l}-2 \mathfrak{R}\left\{\int _ { \Theta } \left[\mathscr{M}_{l}(\Omega) e^{-j \Phi_{l}(\Omega)}\right.\right. \\
& \left.\left.\cdot \sum_{n=1}^{N} \alpha_{n} \tilde{f}_{n}(\Omega) e^{j \psi_{n l}}\right] \mathrm{~d} \Omega\right\}
\end{aligned}
$$

where $\eta_{l}$ is a term independent of $\Phi_{l}(\Omega)$. From this latter expression, the phase function minimizing $\mathscr{I}(\mathbf{a} ; \Phi)$ can be immediately evaluated as

$$
\Phi_{l}(\Omega)=\arg \left[\sum_{n=1}^{N} \alpha_{n} \tilde{f}_{n}(\Omega) e^{j \psi_{n l}}\right]
$$

for $l=1, \ldots, L$.

Therefore, the development of the algorithm can be summarized as follows (Figure 1). Given the array parameters,

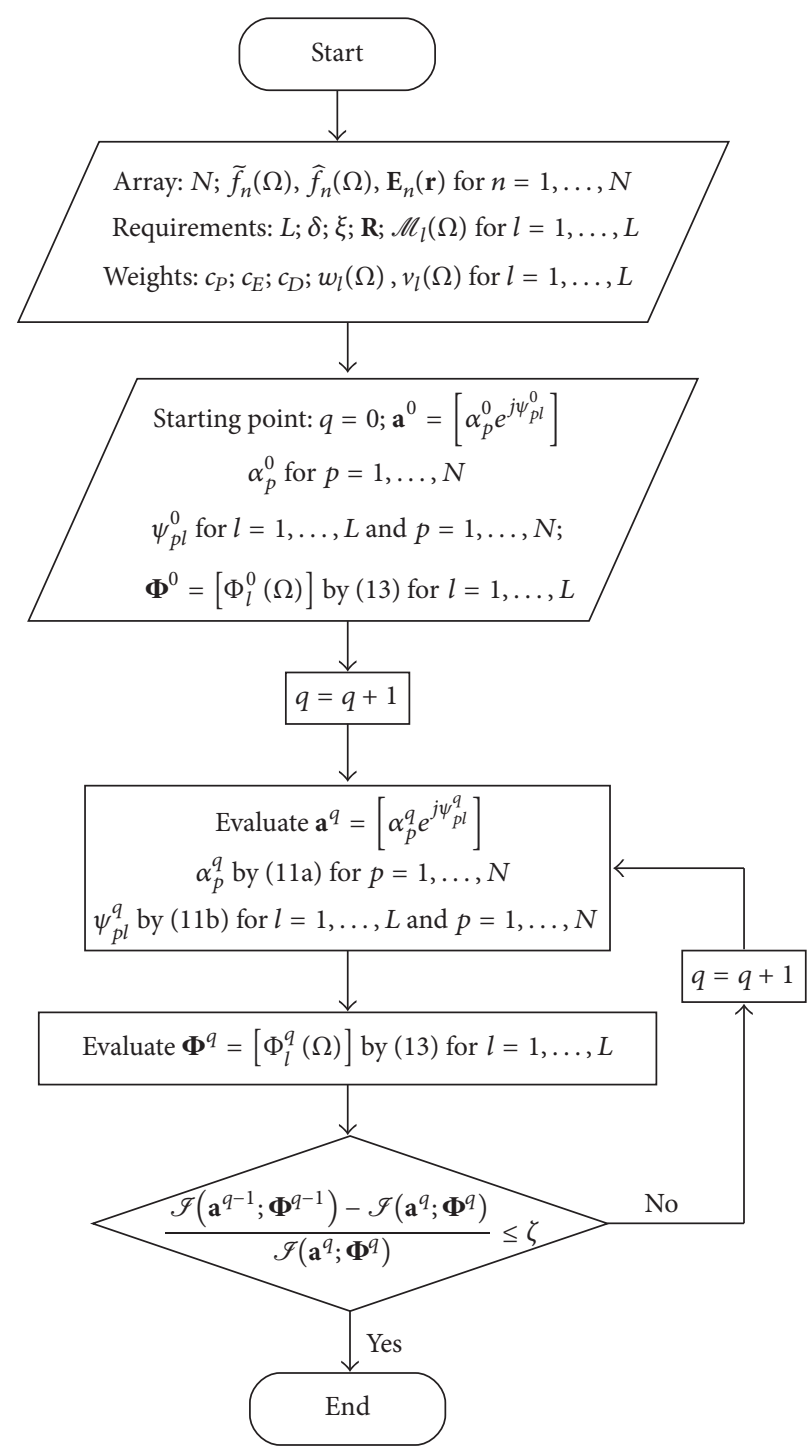

FIGURE 1: Synthesis algorithm.

the synthesis requirements, and the weights for the cost function, the algorithm is initialized (iteration $q=0$ ) by selecting a starting point $\mathbf{a}^{0}$, from which the set of functions $\Phi^{0}$ is evaluated by (13) for $l=1, \ldots, L$. At each successive iteration $q=1,2, \ldots$, two operations are carried out. Firstly, the set of excitations $\mathbf{a}^{q}$ is updated by evaluating (10a), (10b), and (10c), from which one can estimate the amplitudes $\alpha_{p}^{q}$ by (11a) for $p=1, \ldots, N$ and the phases $\psi_{p l}^{q}$ by (11b) for $l=1, \ldots, L$ and $p=1, \ldots, N$. Secondly, the set $\Phi^{q}$ is updated by (13) for $l=1, \ldots, L$. Thus, at each iteration, which substantially consists of two subiterations, (11a), (11b), and (13) minimize $\mathscr{I}(\mathbf{a} ; \boldsymbol{\Phi})$. By consequence, it results in $\mathscr{I}\left(\mathbf{a}^{0} ; \boldsymbol{\Phi}^{0}\right) \geq \mathscr{I}\left(\mathbf{a}^{1} ; \boldsymbol{\Phi}^{1}\right) \geq \cdots \geq \mathscr{I}\left(\mathbf{a}^{q} ; \boldsymbol{\Phi}^{q}\right) \geq \cdots$, and hence the sequence $\left\{\mathscr{I}_{q}\right\}=\left\{\mathscr{I}\left(\mathbf{a}^{q} ; \boldsymbol{\Phi}^{q}\right)\right\}$ is nonincreasing and thus convergent. The iterative procedure may be terminated when $\left(\mathscr{I}_{q-1}-\mathscr{I}_{q}\right) / \mathscr{I}_{q} \leq \zeta$ by selecting a suitable threshold $\zeta$. 


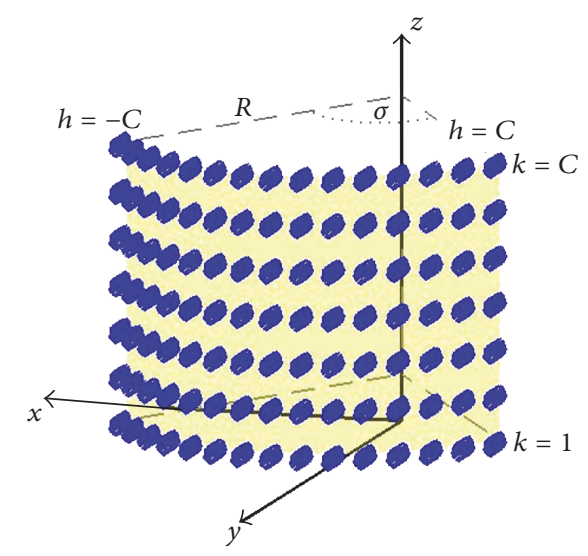

FIGURE 2: Antenna structure for the first application of the proposed algorithm: cylindrical arc array of quadrifilar helix antennas.

There are two main advantages of the presented synthesis approach. The first one consists in the possibility of managing really general synthesis problems, involving $3 \mathrm{D}$ pattern reconfigurability for conformal arrays with near-field, polarization, and DRR requirements. The second advantage is due to the derivation of the closed-form expressions in (11a), (11b), and (13), which allow the update of the excitations at each iteration by a low computational cost.

\section{Numerical Results}

The proposed algorithm is numerically tested considering two different synthesis problems. The first problem involves a conformal array, which is used to manage a scenario where pattern reconfigurability, polarization control, and DRR reduction are jointly required. The second problem relies on the adoption of a three-ring array, which is used to provide pattern reconfigurability, near-field control, and DRR reduction. As discussed in Section 2.1, the mutual coupling effects are taken into account by adopting the technique used in [17]. Accordingly, for each of the two considered antenna structures, the single-element patterns $\widetilde{\mathscr{F}}_{n}(\Omega), \widehat{\mathscr{F}}_{n}(\Omega)$, and $\mathbf{E}_{n}(\mathbf{r})$ in the presence of the rest of the array are evaluated by SuperNEC 2.7 electromagnetic simulator considering a unity-voltage zero-phase feed for the $n$th element and a zerovoltage feed for the other ones. The zenith and azimuth domains are discretized at steps of 4 degrees; hence the desired directions and the angular regions of interest will be identified as multiples of 4 degrees. The method is implemented in Matlab on a Sun Ultra 24 workstation equipped with an Intel Core2 Quad Q9300 processor with $2.50 \mathrm{MHz}$ of clock frequency. Since the algorithm is iterative and so cannot be parallelized, its execution involves a unique core of the processor.

4.1. First Application: Pattern Reconfigurability, Polarization Control, and DRR Reduction. The radiating structure adopted for the first application is a cylindrical arc array of quadrifilar helix antennas (Figure 2) [14]. The arc has aperture $\sigma=90^{\circ}$ and radius $R=4.46 \lambda$, where $\lambda$ denotes the
TABLE 1: First application: overall performance.

\begin{tabular}{lcc}
\hline & $c_{P}=c_{D}=0$ & $c_{P}=c_{D}=20$ \\
\hline$l=1$ : SLL $[\mathrm{dB}]$ & -15.2 & -10.1 \\
$l=1$ : maximum AR in $\Theta_{1}[\mathrm{~dB}]$ & 2.4 & 0.5 \\
$l=2$ : SLL [dB] & -11.8 & -10.4 \\
$l=2$ : maximum AR in $\Theta_{2}[\mathrm{~dB}]$ & 3.5 & 0.5 \\
DRR & 61.8 & 27.3 \\
CPU time [s] & 45.7 & 23.3 \\
\hline
\end{tabular}

wavelength. The generic two-turn helix identified by the pair of integers $(h, k)$ has radius $0.1 \lambda$, turn spacing $0.2 \lambda$, and axis parallel to the $y$-axis and lies in position $R \sin [h \sigma /(2 C)] \widehat{\mathbf{x}}+$ $R \cos [h \sigma /(2 C)] \widehat{\mathbf{y}}+k \lambda / 2 \widehat{\mathbf{z}}$, with $C=7$. Thus, the array has $N=(2 C+1) C=105$ elements.

The developed algorithm is applied to this array to solve a problem involving pattern reconfigurability, polarization control, and DRR reduction. In particular, $L=2$ farfield amplitude patterns with the same distribution of the excitation amplitudes must be synthesized. The first desired pattern $\mathscr{M}_{1}(\Omega)$ (case $l=1$ ) has a SLL of $-10 \mathrm{~dB}$, the maximum at $\left[88^{\circ}, 80^{\circ}\right]$. The second desired pattern $\mathscr{M}_{2}(\Omega)$ (case $l=2$ ) has a SLL of $-10 \mathrm{~dB}$, the maximum at $\left[92^{\circ}, 96^{\circ}\right]$, and presents a $2 \mathrm{D}$ null region $\left[82^{\circ}, 96^{\circ}\right] \times\left[-88^{\circ},-56^{\circ}\right]$ in which the synthesized pattern must be lower than $-30 \mathrm{~dB}$. The desired radiated far-field vector must be right-hand circularly polarized $(\delta=1, \xi=-\pi / 2)$ within the $3 \mathrm{~dB}$ main lobe of each of the two synthesized patterns. Thus, the problem considers both beam and null synthesis and requires that a high level of polarization purity is maintained when switching from one pattern to another. Furthermore, these objectives should be achieved by a reduced DRR to simplify the feeding network.

For this application the algorithm is run by setting $c_{E}=0$, $\zeta=10^{-6}, \boldsymbol{\alpha}^{0}=\mathbf{0}_{N \times 1}, \psi^{0}=\mathbf{0}_{N \times L}, w_{l}(\Omega)=\left[\mathscr{M}_{l}(\Omega)\right]^{-\mu}$ with $\mu=6$, and $v_{l}(\Omega)=10^{\chi}$ with $\chi=5$ for $l=1,2$. The obtained results are shown in Figure 3, which reports the $2 \mathrm{D}$ cuts corresponding to the direction of maximum radiation for both synthesized patterns, and in Table 1, which 

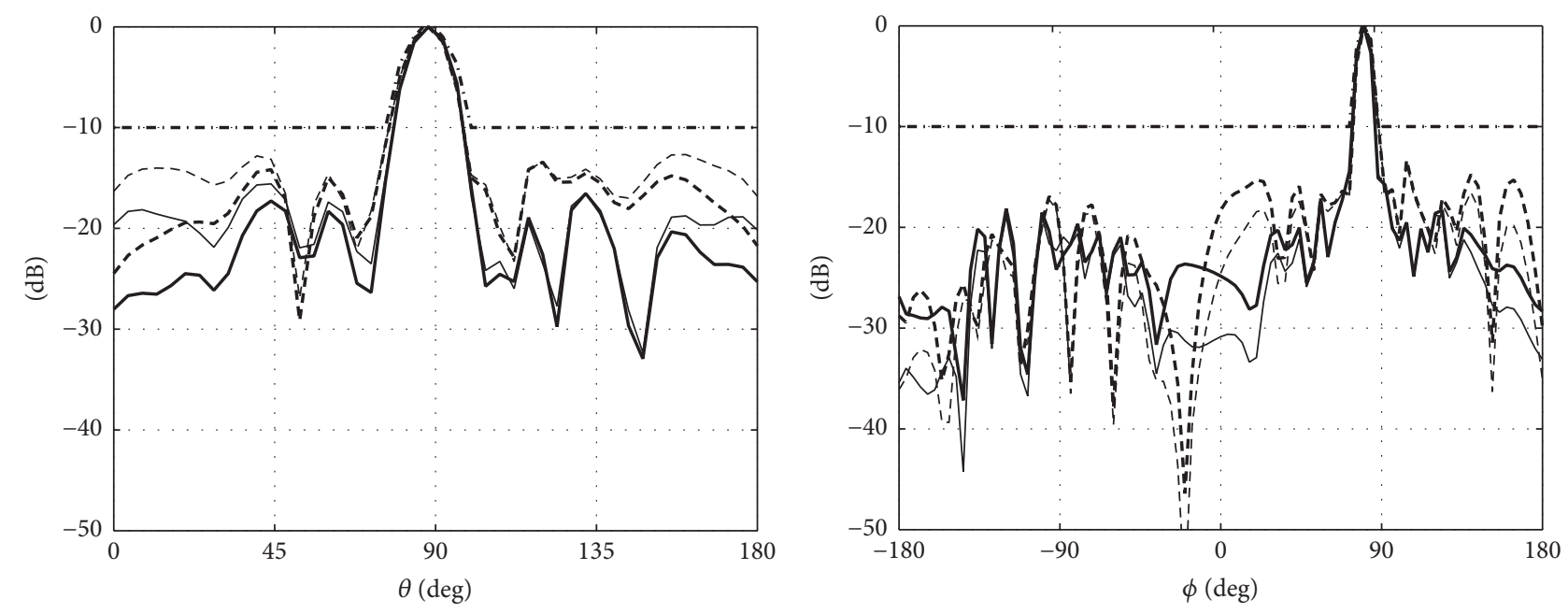

$$
\begin{array}{ll}
\cdots M_{1} & \cdots\left|F_{\theta}\right|^{2}\left(c_{P}=c_{D}=20\right) \\
-\left|F_{\theta}\right|^{2}\left(c_{P}=c_{D}=0\right) & --\left|F_{\phi}\right|^{2}\left(c_{P}=c_{D}=20\right) \\
-\left|F_{\phi}\right|^{2}\left(c_{P}=c_{D}=0\right) &
\end{array}
$$

(a)

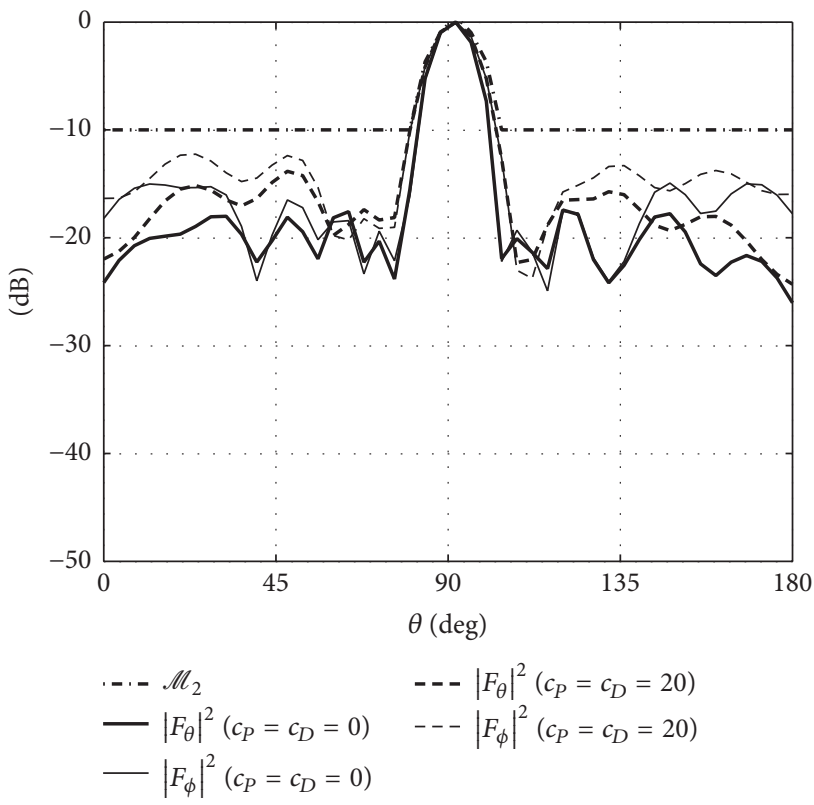

(c)

$$
\begin{array}{ll}
\cdots \mathscr{M}_{1} & --\left|F_{\theta}\right|^{2}\left(c_{P}=c_{D}=20\right) \\
-\left|F_{\theta}\right|^{2}\left(c_{P}=c_{D}=0\right) & ---\left|F_{\phi}\right|^{2}\left(c_{P}=c_{D}=20\right) \\
-\left|F_{\phi}\right|^{2}\left(c_{P}=c_{D}=0\right) &
\end{array}
$$

(b)

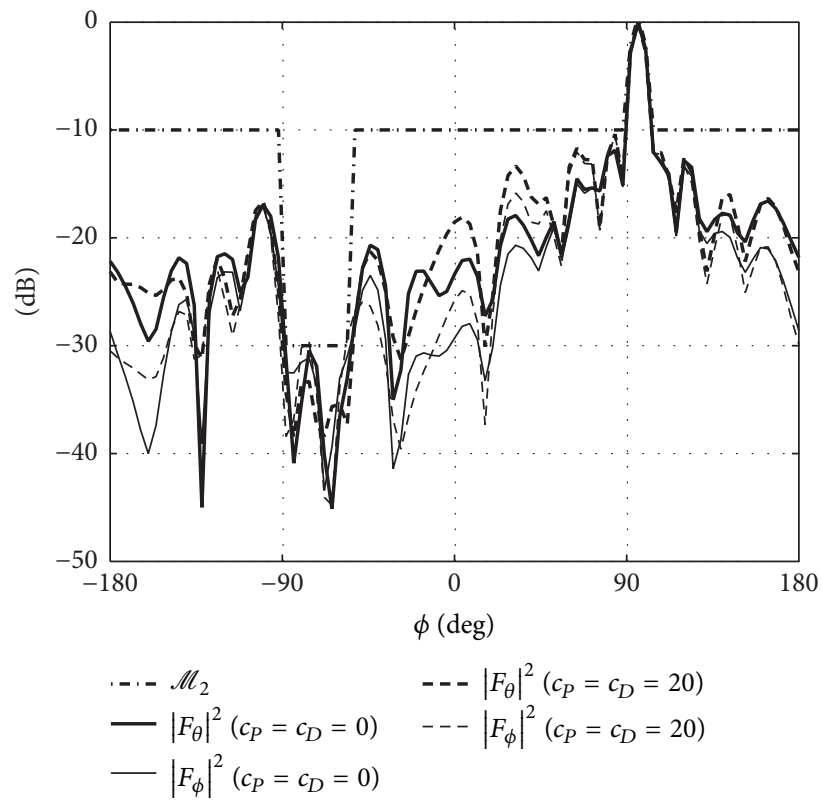

(d)

FIGURE 3: First application, 2D far-fields pattern cuts: (a) $l=1$ (first pattern), cut at $\phi=80^{\circ}$, (b) $l=1$ (first pattern), cut at $\theta=88^{\circ}$, (c) $l=2$ (second pattern), cut at $\phi=96^{\circ}$, and (d) $l=2$ (second pattern), cut at $\theta=92^{\circ}$.

reports the SLL referring to the entire angular domain $\Theta$, the maximum Axial Ratio (AR) in the $3 \mathrm{~dB}$ main lobe of each synthesized pattern, the DRR, and the CPU time required for the synthesis. With reference to these results, we may first notice that, in all the considered cases, the synthesized patterns properly approximate the desired ones, even in the presence of null constraints. A direct comparison between the case $c_{P}=c_{D}=0$ (just reconfigurability) and the case $c_{P}=c_{D}=20$ (reconfigurability, polarization control, and DRR reduction) reveals that, using the proposed algorithm, the pattern reconfigurability can be maintained simultaneously improving the purity of the circular polarization and halving the DRR of the excitations. Moreover, the developed approach is considerably fast, since the CPU time necessary for the synthesis resulted in being lower than a minute for both cases.

4.2. Second Application: Pattern Reconfigurability, Near-Field, and DRR Reduction. The radiating structure adopted for the 


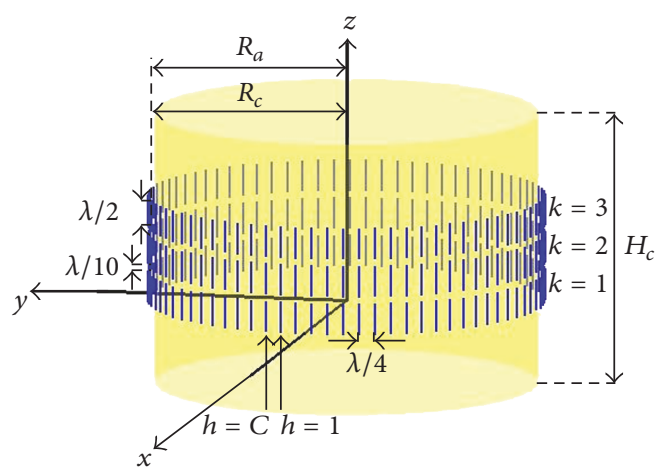

FIGURE 4: Antenna structure for the second application of the proposed algorithm: three-ring array of center-fed half-wavelength dipole antennas placed around a perfectly conducting cylinder.

TABLE 2: Second application: overall performance.

\begin{tabular}{lcc}
\hline & $c_{E}=c_{D}=0$ & $c_{E}=c_{D}=10$ \\
\hline$l=1:$ SLL $[\mathrm{dB}]$ & -13.5 & -11.3 \\
$l=1:\left|\mathbf{E}\left(\mathbf{a}_{1} ; \mathbf{r}\right)\right|_{\max }$ in $\mathbf{R}[\mathrm{dB}]$ & -43.5 & -53.1 \\
$l=2:$ SLL $[\mathrm{dB}]$ & -19.5 & -14.5 \\
$l=2:\left|\mathbf{E}\left(\mathbf{a}_{2} ; \mathbf{r}\right)\right|_{\max }$ in $\mathbf{R}[\mathrm{dB}]$ & -44.0 & -54.5 \\
DRR & 385.4 & 30.2 \\
CPU time $[\mathrm{s}]$ & 52.7 & 34.7 \\
\hline
\end{tabular}

second application is a three-ring array of center-fed halfwavelength dipoles placed around a perfectly conducting cylinder with axis coincident with the $z$-axis (Figure 4) [17]. The cylinder has radius $R_{c}=3 \lambda$ and height $H_{c}=4.3 \lambda$. The center of the $h$ th dipole of the $k$ th ring, which has $C=78$ elements and radius $R_{a}=3.1 \lambda$, lies in position $R_{a} \cos (2 \pi h / C) \widehat{\mathbf{x}}+R_{a} \sin (2 \pi h / C) \widehat{\mathbf{y}}+[3(k-1) / 5+1 / 4] \lambda \widehat{\mathbf{z}}$. Hence, the array has $N=3 C=234$ elements.

The algorithm is applied to this array to solve a problem involving pattern reconfigurability with $L=2$, near-field, and DRR reduction. Both desired patterns have a SLL of $-13 \mathrm{~dB}$, the maximum at $\left[88^{\circ}, 0^{\circ}\right]$, and a $2 \mathrm{D}$ null region $\left[82^{\circ}, 96^{\circ}\right] \times$ $\left[-140^{\circ},-100^{\circ}\right]$ in which the synthesized pattern must be lower than $-40 \mathrm{~dB}$. The first desired pattern $\mathscr{M}_{1}(\Omega)$ (case $l=1$ ) has a narrow azimuth beam with a $3 \mathrm{~dB}$ beamwidth of $4^{\circ}$, while the second desired pattern $\mathscr{M}_{2}(\Omega)$ (case $l=2$ ) has a wide azimuth beam with a $3 \mathrm{~dB}$ beamwidth of $32^{\circ}$. Beside the reconfigurability requirement, the DRR must be limited and the electric near-field amplitude must be reduced in the $3 \mathrm{D}$ space region $\mathbf{R}=\{(x, y, z) \in[6 \lambda, 10 \lambda] \times[-2 \lambda, 2 \lambda] \times[0, \lambda]\}$.

For this application the algorithm is run setting $c_{P}=0$, $\zeta=10^{-4}, \boldsymbol{\alpha}^{0}=\mathbf{0}_{N \times 1}, \psi^{0}=\mathbf{0}_{N \times L}, w_{l}(\Omega)=\left[\mathscr{M}_{l}(\Omega)\right]^{-\mu}$ with $\mu=3.5$, and $u_{l}(\mathbf{r})=10^{\varsigma}$ with $\varsigma=2$ for $l=1,2$. The region $\mathbf{R}$ is discretized with a dense regular grid of step $\lambda / 8$, in order to obtain an accurate estimation of the near-field behavior. Adopting these settings, one obtains Figure 5, which presents the $2 \mathrm{D}$ cuts in the direction of maximum radiation for the two synthesized patterns, Figure 6, which shows the contour plots of the 2D cuts of the electric near-field amplitude, and Table 2, which reports the SLL referring to the entire angular domain $\Theta$, the maximum electric near-field amplitude in the region $\mathbf{R}$ for each synthesized pattern, the DRR, and the CPU time. In particular, Figure 6 refers to the worst case, that is, the case corresponding to the 2D cut (among those parallel to the $x$ - $y$ plane) and the electric near-field (among $\left|\mathbf{E}\left(\mathbf{a}_{1} ; \mathbf{r}\right)\right|$ and $\left.\left|\mathbf{E}\left(\mathbf{a}_{2} ; \mathbf{r}\right)\right|\right)$ in which the lowest amplitude reduction is achieved. Concerning this aspect, one may observe that, even in this worst case, a near-field reduction in the order of $10 \mathrm{~dB}$ is guaranteed while maintaining the far-field patterns close to the desired ones. Moreover, selecting $c_{E}=c_{D}=10$, the DRR can be reduced more than twelve times with respect to the case $c_{E}=c_{D}=0$, thus making the realization of the array feeding network more feasible. Furthermore, also in this second application, the CPU time required for the synthesis remained lower than one minute, hence confirming that the proposed algorithm is fast even when arrays with more than two hundred elements have to be managed.

\section{Conclusions}

An iterative method for the 3D synthesis of conformal antenna arrays able to account for multiple requirements has been presented. The algorithm relies on the reformulation of an original field synthesis problem into a power synthesis one with the aim of deriving closed-form expressions for 


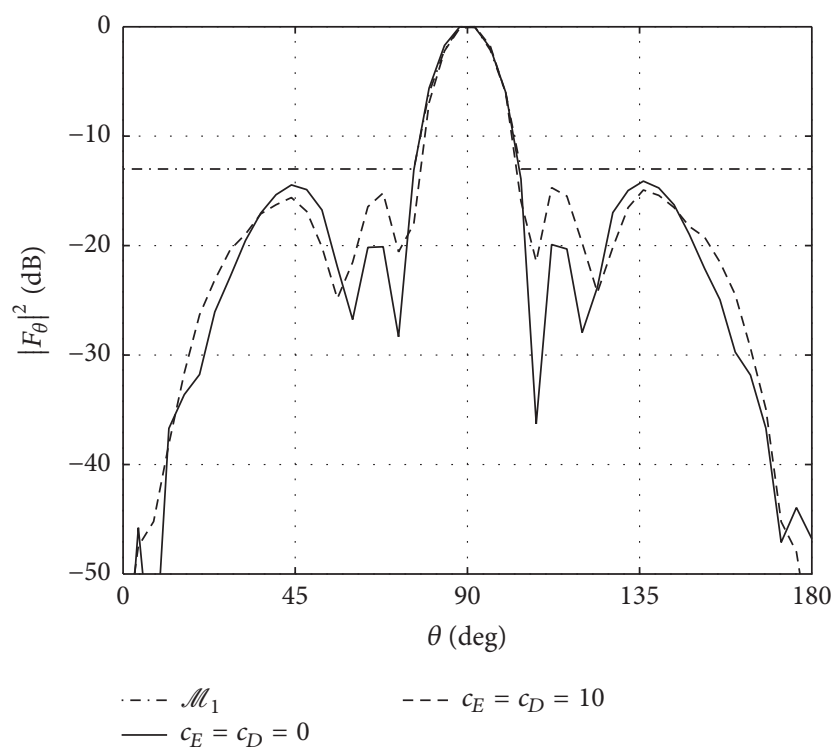

(a)

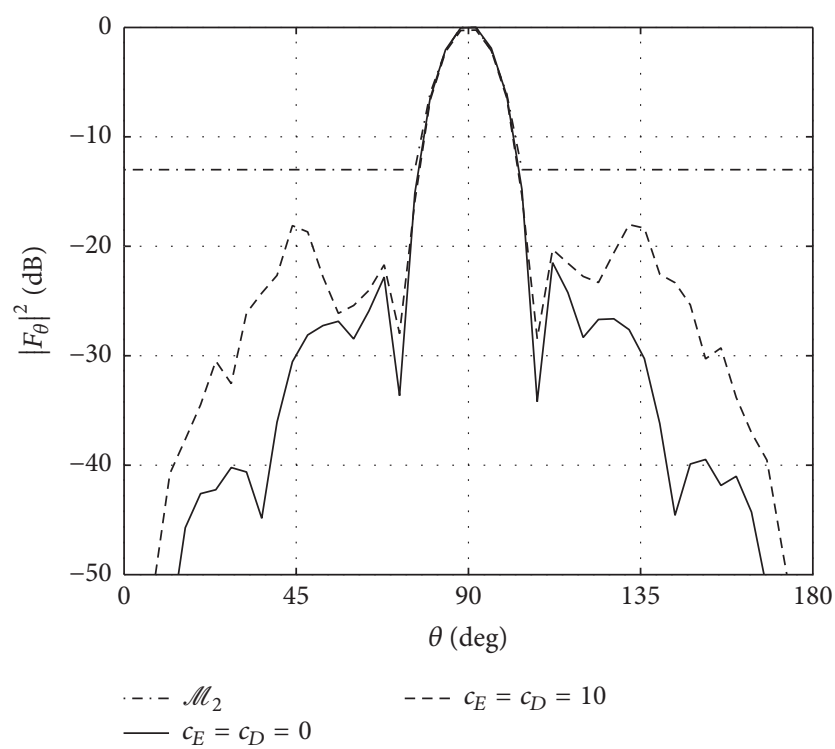

(c)

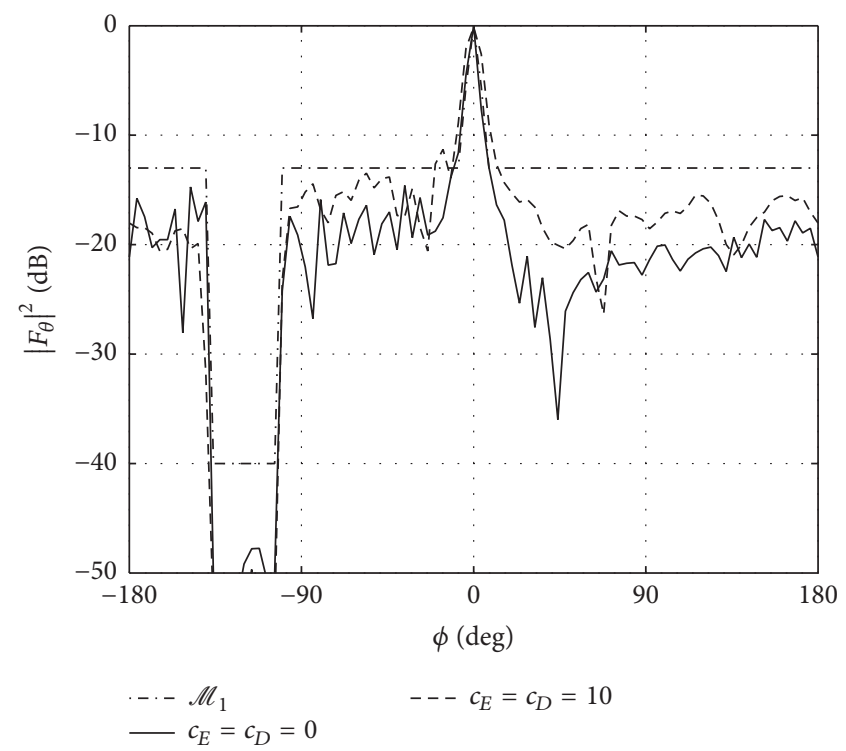

(b)

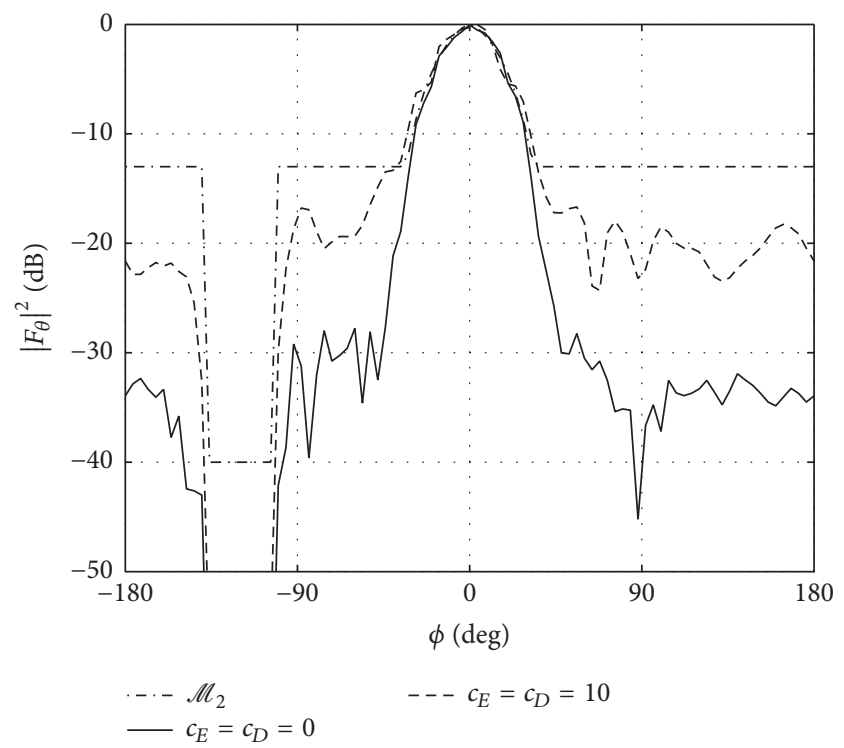

(d)

Figure 5: Second application, 2D far-field pattern cuts: (a) $l=1$ (first pattern), cut at $\phi=0^{\circ}$, (b) $l=1$ (first pattern), cut at $\theta=88^{\circ}$, (c) $l=2$ (second pattern), cut at $\phi=0^{\circ}$, and (d) $l=2$ (second pattern), cut at $\theta=88^{\circ}$.

the calculations carried out at each iteration. The conceived solution has been validated by considering different conformal structures and realistically taking into account the mutual coupling between the array elements. The reported applications have confirmed the considerable versatility of the developed method, which is able to manage really different scenarios, characterized by requirements on the farfield pattern, including reconfigurability and null synthesis, and constraints on the polarization, the DRR, and the electric near-field amplitude in a given region close to the antenna. The obtained results have also put into evidence that the algorithm is very fast, since the solution of all the considered synthesis problems has required less than one minute.

\section{Conflicts of Interest}

The authors declare that there are no conflicts of interest regarding the publication of this paper.

\section{Acknowledgments}

This work is partly supported by the Italian Ministry of University and Research (MIUR) within the project FRA 


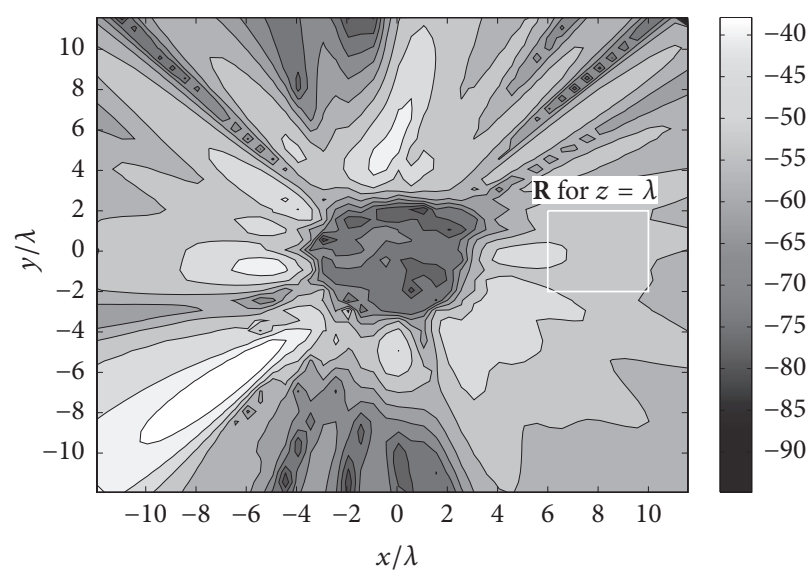

(a)

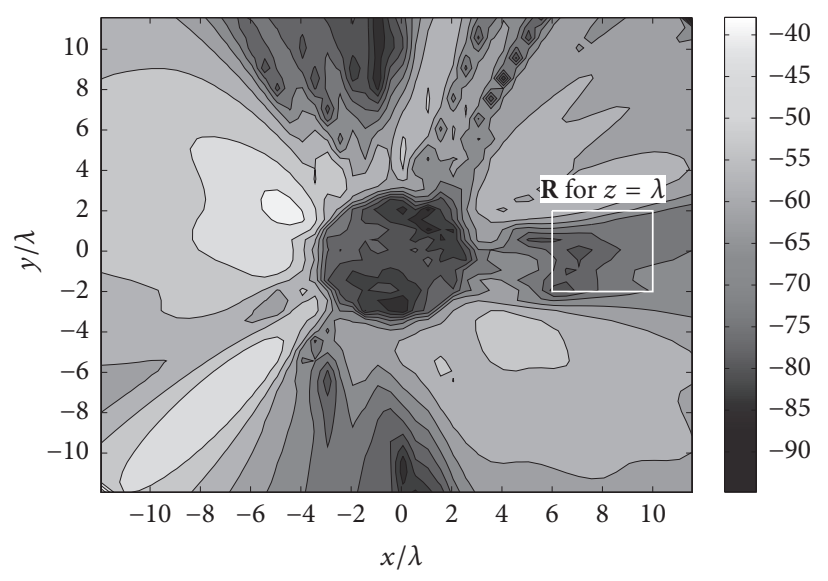

(b)

Figure 6: Second application, contour plots of the 2D cuts of the electric near-field amplitude in the worst case (|E( $\left.\mathbf{a}_{1} ; \mathbf{r}\right) \mid$ for $\left.z=\lambda\right)$ : (a) $c_{E}=c_{D}=0 ;(\mathrm{b}) c_{E}=c_{D}=10$.

2015 (University of Trieste, Italy), entitled "Peer-to-Peer Millimeter-Wave Communications in 5G Networks: Theoretical Modeling and Algorithms for Massive MIMO Systems.”

\section{References}

[1] Y. I. Choni, "Synthesis of an antenna according to a given amplitude radiation pattern, AIEE Radio Engineering and Electronic Physics," Phys, vol. 16, pp. 770-778, May 1971.

[2] J. A. Rodríguez, L. Landesa, J. L. Rodríguez, F. Obelleiro, F. Ares, and A. García-Pino, "Pattern synthesis of array antennas with arbitrary elements by simulated annealing and adaptive array theory," Microwave and Optical Technology Letters, vol. 20, no. 1, pp. 48-50, 1999.

[3] L. I. Vaskelainen, "Constrained least-squares optimization in conformal array antenna synthesis," Institute of Electrical and Electronics Engineers. Transactions on Antennas and Propagation, vol. 55, no. 3, part 2, pp. 859-867, 2007.

[4] O. M. Bucci, G. Franceschetti, G. Mazzarella, and G. Panariello, "Intersection approach to array pattern synthesis," IEE Proceedings H: Microwaves, Antennas and Propagation, vol. 137, no. 6, pp. 349-357, 1990.

[5] O. M. Bucci, G. D'Elia, and G. Romito, "Power synthesis of conformal arrays by a generalised projection method," IEE Proceedings: Microwaves, Antennas and Propagation, vol. 142, no. 6, pp. 467-471, 1995.

[6] R. Gholami, B. Zakeri, H. Abedi, and S. Mohseni, "Reduction of dynamic range ratio through competition over resources to synthesize planar array antennas," AEU-International Journal of Electronics and Communications, vol. 70, no. 11, pp. 1522-1531, 2016.

[7] A. D. Khzmalyan and A. S. Kondrat'yev, "Phase-only synthesis of antenna array amplitude pattern," International Journal of Electronics, vol. 81, no. 5, pp. 585-589, 1996.

[8] M. Mouhamadou, P. Vaudon, and M. Rammal, "Smart antenna array patterns synthesis: null steering and multi-user beamforming by phase control," Progress in Electromagnetics Research, vol. 60, pp. 95-106, 2006.

[9] M. Comisso and R. Vescovo, "Fast power synthesis for reconfigurable arrays with dynamic range ratio reduction," in Proceedings of the 13th International Conference on Electromagnetics in
Advanced Applications (ICEAA '11), pp. 374-377, Torino, Italy, September 2011.

[10] G. Buttazzoni and R. Vescovo, "An efficient and versatile technique for the synthesis of 3D copolar and crosspolar patterns of phase-only reconfigurable conformal arrays with DRR and near-field control," Institute of Electrical and Electronics Engineers. Transactions on Antennas and Propagation, vol. 62, no. 4, part 1, pp. 1640-1651, 2014.

[11] W. Bu-hong and G. Ying, "Frequency-invariant and low crosspolarization pattern synthesis for conformal array antenna," in Proceedings of the IEEE Radar Conference (RADAR '08), pp. 1-6, Rome, Italy, 2008.

[12] R. K. Baee, K. Forooraghi, and S. Chamaani, "Conformal array pattern synthesis using a hybrid WARP/2LB-MOPSO algorithm," International Journal of Antennas and Propagation, vol. 2012, Article ID 202906, 7 pages, 2012.

[13] B. Fuchs and J. J. Fuchs, "Optimal polarization synthesis of arbitrary arrays with focused power pattern," Institute of Electrical and Electronics Engineers. Transactions on Antennas and Propagation, vol. 59, no. 12, pp. 4512-4519, 2011.

[14] M. Comisso and R. Vescovo, "Fast 3D pattern synthesis with polarization and dynamic range ratio control for conformal antenna arrays," International Journal of Antennas and Propagation, vol. 2014, Article ID 106741, 9 pages, 2014.

[15] L. Landesa, F. Obelleiro, J. L. Rodríguez, J. A. Rodríguez, F. Ares, and A. G. Pino, "Pattern synthesis of array antennas with additional isolation of near field arbitrary objects," Electronics Letters, vol. 34, no. 16, pp. 1540-1542, 1998.

[16] H. Steyskal, "Synthesis of antenna patterns with imposed nearfield nulls," Electronics Letters, vol. 42, no. 19, pp. 1079-1080, 2006.

[17] M. Comisso and R. Vescovo, "3D power synthesis with reduction of near-field and dynamic range ratio for conformal antenna arrays," IEEE Transactions on Antennas and Propagation, vol. 59, no. 4, pp. 1164-1174, 2011.

[18] G. Buttazzoni and R. Vescovo, "Power synthesis for reconfigurable arrays by phase-only control with simultaneous dynamic range ratio and near-field reduction," IEEE Transactions on Antennas and Propagation, vol. 60, no. 2, pp. 1161-1165, 2012. 


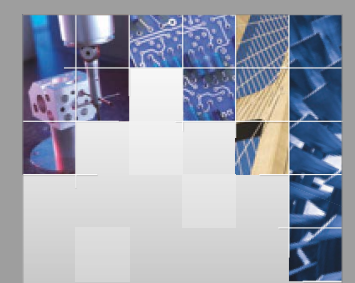

\section{Enfincering}
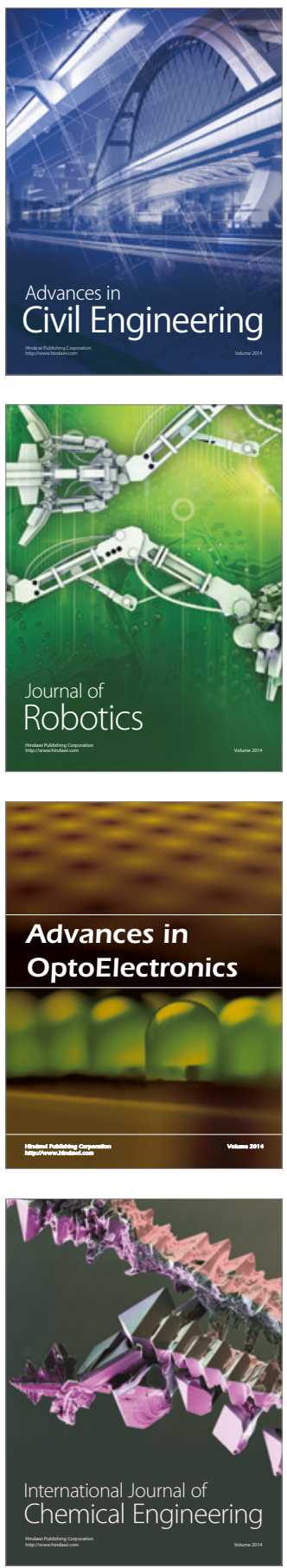

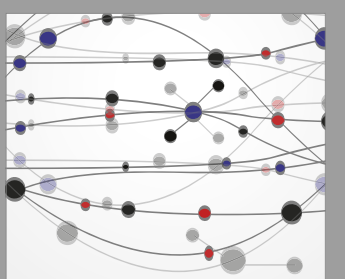

The Scientific World Journal

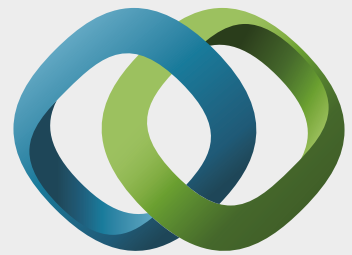

\section{Hindawi}

Submit your manuscripts at

https://www.hindawi.com
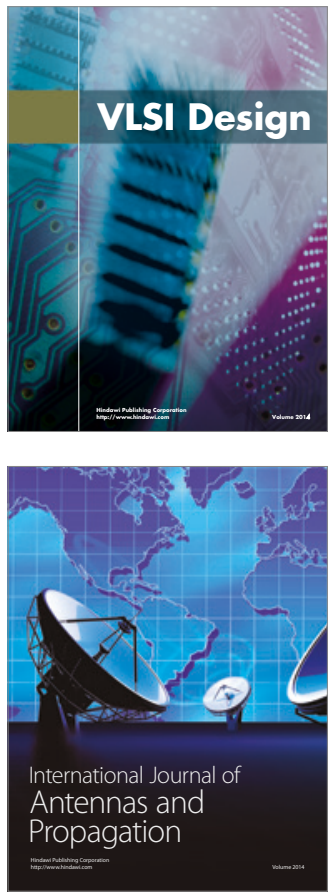

\section{Rotating}

Machinery
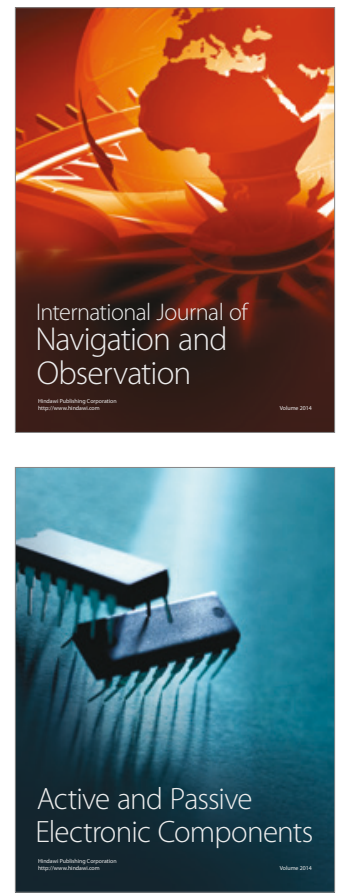
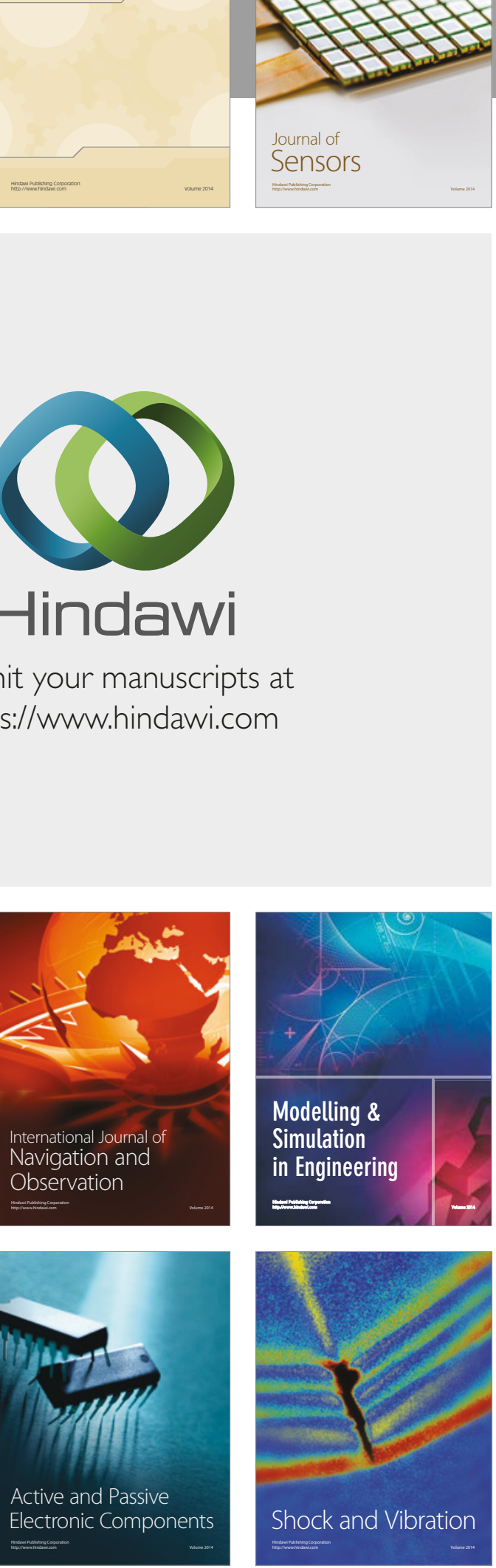
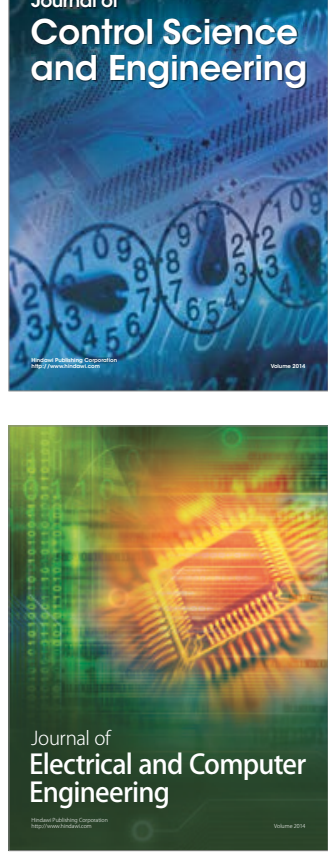

Distributed

Journal of

Control Science

and Engineering
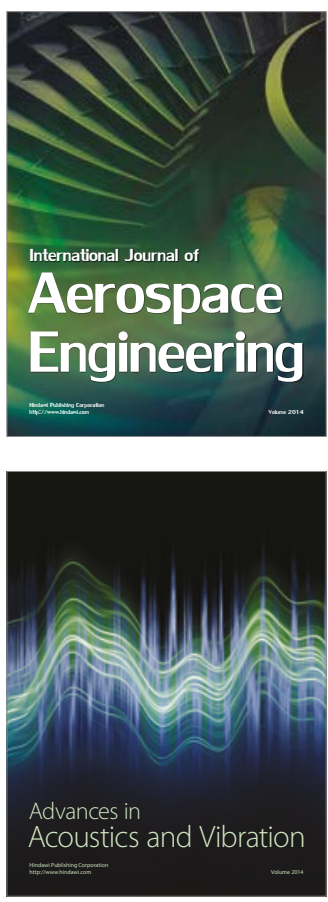

Sensor Networks 